\title{
Juvenile Idiopathic Arthritis: An Idea Whose Time Has Gone?
}
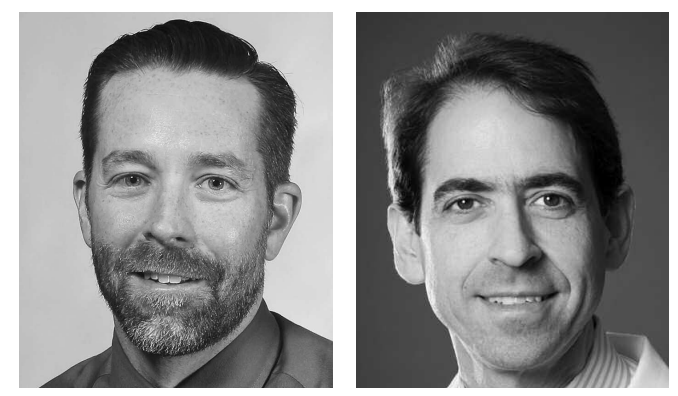

The development of the International League of Associations for Rheumatology (ILAR) classification of juvenile idiopathic arthritis (JIA) in the 1990s represented a significant change in thinking about childhood-onset arthritis. It combined the similar but disparate concepts of juvenile rheumatoid arthritis and juvenile chronic arthritis to create mutually exclusive categories of JIA ${ }^{1}$. Always intended as a stepping stone rather than as a final categorization schema, the weaknesses of the ILAR classification are by now well documented, including lack of validation, overly restrictive exclusion criteria, and inclusion criteria often not assessed clinically.

Clearly, creating disease nomenclature is difficult. An ideal classification system brings together disorders that share key elements of pathophysiology, clinical features, prognosis, and response to treatment. In this issue of The Journal, Professor Alberto Martini and colleagues continue the quest to improve the categorization of childhood-onset arthritis ${ }^{2}$. Under the sponsorship of the Pediatric Rheumatology International Trials Organization (PRINTO), an international group of 13 senior pediatric rheumatologists applied established consensus methodologies to propose new JIA classification criteria. The results are predictably controversial.

Perhaps most puzzling is the proposal that JIA, despite its name, is no longer a collection of arthritides but rather simply "a group of inflammatory disorders that begins before the 18th birthday and persists for at least 6 weeks." There is little here to distinguish JIA from all other chronic inflammatory diseases of childhood. The motivation is to keep systemic arthritis within JIA while dropping the requirement for arthritis (more on this later). They preserve "JIA" because the US Food and Drug Administration and the European Medicines Agency use the term in product labeling, a plausible rationale, although neither agency strictly adheres to ILAR nomenclature. Reasonable alternatives would have been to allow systemic JIA to have an arthritis exception or to remove it from JIA entirely.

The authors do propose a major step forward, one long overdue: they eliminate the distinction between oligoarthritis and polyarthritis, referring to whether a child has fewer than 5 versus 5 or more affected joints in the first 6 months of disease. This cutoff was never supported by evidence, was difficult to apply because disease onset often cannot be dated accurately, and is now largely uninterpretable in the face of early, effective disease-modifying therapy.

Martini and colleagues also acknowledge that many childhood-onset arthritis phenotypes have adult counterparts, referencing a "growing impetus to harmonize" the criteria for disorders across the spectrum of age of onset ${ }^{2}$. Unfortunately, the proposed nomenclature does little to accomplish this aim.

For example, they note that rheumatoid factor-positive childhood arthritis is indeed synonymous with seropositive RA, a belief now convincingly supported by genetic evidence ${ }^{3,4,5}$. Nevertheless, the authors then assign a new name, rheumatoid factor-positive JIA, ${ }^{2}$ and do not consider rheumatoid arthritis classification criteria jointly developed by the American College of Rheumatology and the European League Against Rheumatism ${ }^{6}$.

The sharp division between pediatric and adult arthritis is now old enough that most rheumatologists take it for granted, but in fact it should strike us as remarkable and even bizarre $^{3}$. We do not divide asthma, diabetes, or inflammatory bowel disease by age of onset, even if children and adults sometimes present differently and can require different management. In the rheumatic diseases, early presentation typically correlates with greater genetic risk, and often with greater severity, but it is important that we do not confuse such variation to represent different disorders, as suggested by assignment of different names.

We also agree with the authors' assertion that systemic JIA is the same as adult-onset Still disease (AOSD), an idea for which there is extensive support ${ }^{3}$. They incorporate concepts from the Yamaguchi criteria for $\mathrm{AOSD}^{7}$, most notably the lack of requirement for arthritis, although features such as sore throat, liver enzyme elevation, and leukocytosis threshold are omitted or modified on the basis of expert opinion. The resulting hybrid classification system

See PRINTO JIA classification criteria, page 190

Personal non-commercial use only. The Journal of Rheumatology Copyright (C) 2019. All rights reserved. 
remains to be tested. The authors considered the term Still disease, choosing instead to remain with systemic JIA to avoid eponyms, but in doing so they again maintain the arbitrary division between pediatric and adult disease.

Systemic JIA provides a good example of why terminology matters. The adult rheumatology community has been seemingly slower to adopt interleukin (IL)-1 and IL-6 blockade for AOSD. Would this have been so if both diseases were called Still disease? We have gleaned much about treating childhood-onset arthritis from adult clinical trials. Perhaps if we harmonized our nomenclature, we could speed the application of generalizable knowledge and even return the favor by allowing adult patients to benefit from pediatric expertise.

Regarding enthesitis-related arthritis (ERA), there is a similar missed opportunity. This disorder is likened to the adult spondyloarthropathies (SpA), but the new name enthesitis/spondylitis-related JIA - perhaps even less comprehensible to families than ERA - again divides children from adults. Once more, no reference is made to classification criteria developed for adults $8,9,10$.

Finally, Martini and colleagues propose a new pediatric-specific arthritis: early-onset ANA-positive JIA, defined as arthritis beginning before the seventh birthday and accompanied by a positive antinuclear antibody (ANA) at a titer of $\geq 1: 160$, twice at least 3 months apart ${ }^{2}$. This suggestion derives from 2 series that found ANA positivity to correlate with early age of onset, female skew, uveitis risk, and certain features of clinical course ${ }^{11,12}$. A histological study found synovial tissues from children with ANA at this titer to have more lymphoid aggregates than those without ${ }^{13}$. Yet ANA-based categorization is still regarded by many with skepticism. ANA titers can vary widely, both with time and with method of ascertainment. Young children are at risk of uveitis even if ANA-negative. None of the published reports tested whether the apparent predictive value of ANA was driven largely by age of onset, as suggested by gene expression data ${ }^{14}$. ANA has not consistently been found to represent an independent predictor of clinical course ${ }^{15,16,17}$. These considerations do not reject the hypothesis that ANA marks a unique subset of arthritis in children, but they do suggest that basing a nomenclature upon this foundation remains premature.

By contrast, like seropositive RA, the SpA, and AOSD, psoriatic arthritis (PsA) is well established in adult rheumatology. The authors' relegation of PsA to the "other" category to be studied further is therefore notable ${ }^{2}$. Arthritis in young children with psoriasis or psoriatic features may manifest differently from that found in adults, posing clear diagnostic and nomenclature challenges ${ }^{18,19,20}$. Yet it strains the credibility of the nomenclature that it offers no way to recognize classic PsA, for example using adult criteria, even as the age of inclusion into JIA is extended officially to the 18th birthday ${ }^{21}$.
Beyond concerns about the individual definitions proposed, there remain more general concerns about process. Classification of childhood-onset arthritis using routine clinical and laboratory measures allows for easy application, and beginning the process with expert opinion is a time-honored tradition. Yet it is unclear whether this will produce substantial advancements in the end. It would seem more appropriate to allow data from biomarkers (including genetics) and response to therapies to help drive the modern classification process.

Underlying all these criticisms remains the larger question of whether JIA remains a useful concept at all. Historically, the division of chronic arthritides by age of onset served a useful purpose. Pediatric rheumatology was a young and struggling specialty, and the JIA nomenclature helped pediatric rheumatologists think carefully about their patients and communicate their observations with each other. The age cutoff at the 16th birthday was an arbitrary but useful boundary for our working definitions. Now that pediatric rheumatology has firmly established itself and is moving on to the bigger task of identifying biological subgroups that respond to specific therapeutics, the utility of eminence-based classification and even the JIA concept itself is rapidly dwindling.

The expression "don't throw the baby out with the bathwater" dates back to the 16th century writings of Thomas Murner. It reminds us not to discard that which is valuable when disposing of that which is no longer useful. Nevertheless, it is sometimes best to acknowledge when a concept that was previously worthwhile is no longer so. The assumption that childhood-onset arthritis is fundamentally different from adult arthritis was never based on evidence, and genetic and biological data increasingly reveal far more similarities than differences ${ }^{3}$. Perhaps it is premature to discard the JIA concept altogether, because the dream of a biology-based arthritis classification remains distant. In the meantime, for research and for clinical practice, we need ways to talk about groups of patients. Martini and colleagues contribute helpful suggestions for reform, the fruit of many decades of careful observation ${ }^{2}$. While we are not ready to fully embrace the result, we recognize that the attempt to validate these proposed revisions will likely increase our understanding of childhood-onset arthritis.

TIMOTHY BEUKELMAN, MD, MSCE, Associate Professor of Pediatrics, Division of Rheumatology, Department of Pediatrics, University of Alabama at Birmingham, Birmingham, Alabama;

PETER A. NIGROVIC, ${ }_{\mathrm{MD}}$,

Associate Professor of Medicine, Brigham and Women's Hospital, Boston Children's Hospital, Harvard Medical School, Boston, Massachusetts, USA.

Personal non-commercial use only. The Journal of Rheumatology Copyright @ 2019 . All rights reserved. 
Address correspondence to Dr. T. Beukelman, The University of Alabama at Birmingham, Division of Pediatric Rheumatology, 1600 7th Ave. South, CPP 210, Birmingham, Alabama 35233-1711, USA.

E-mail: tbeukelman@peds.uab.edu

\section{REFERENCES}

1. Petty RE, Southwood TR, Manners P, Baum J, Glass DN, Goldenberg $\mathrm{J}$, et al. International League of Associations for Rheumatology classification of juvenile idiopathic arthritis: second revision, Edmonton, 2001. J Rheumatol 2004;31:390-2.

2. Martini A, Ravelli A, Avcin T, Beresford MW, Burgos-Vargas R, Cuttica R, et al, for the Pediatric Rheumatology International Trials Organization (PRINTO). Toward new classification criteria for juvenile idiopathic arthritis: step 1, Pediatric Rheumatology International Trials Organization (PRINTO) International Consensus. J Rheumatol 2019;46:190-7.

3. Nigrovic PA, Raychaudhuri S, Thompson SD. Review: Genetics and the classification of arthritis in adults and children. Arthritis Rheumatol 2018;70:7-17.

4. Hinks A, Bowes J, Cobb J, Ainsworth HC, Marion MC, Comeau $\mathrm{ME}$, et al. Fine-mapping the MHC locus in juvenile idiopathic arthritis (JIA) reveals genetic heterogeneity corresponding to distinct adult inflammatory arthritic diseases. Ann Rheum Dis 2016;76:765-72.

5. Hinks A, Marion MC, Cobb J, Comeau ME, Sudman M, Ainsworth $\mathrm{HC}$, et al. Brief report: the genetic profile of rheumatoid factor-positive polyarticular juvenile idiopathic arthritis resembles that of adult rheumatoid arthritis. Arthritis Rheumatol 2018; 70:957-62.

6. Aletaha D, Neogi T, Silman AJ, Funovits J, Felson DT, Bingham CO 3rd, et al. 2010 rheumatoid arthritis classification criteria: an American College of Rheumatology/European League Against Rheumatism collaborative initiative. Ann Rheum Dis 2010; 69:1580-8.

7. Yamaguchi M, Ohta A, Tsunematsu T, Kasukawa R, Mizushima Y, Kashiwagi H, et al. Preliminary criteria for classification of adult Still's disease. J Rheumatol 1992;19:424-30.

8. Rudwaleit M, Landewe R, van der Heijde D, Listing J, Brandt J, Braun J, et al. The development of Assessment of SpondyloArthritis international Society classification criteria for axial spondyloarthritis (part I): classification of paper patients by expert opinion including uncertainty appraisal. Ann Rheum Dis 2009;68:770-6.

9. Rudwaleit M, van der Heijde D, Landewe R, Listing J, Akkoc N, Brandt J, et al. The development of Assessment of
SpondyloArthritis international Society classification criteria for axial spondyloarthritis (part II): validation and final selection. Ann Rheum Dis 2009;68:777-83.

10. Taurog JD, Chhabra A, Colbert RA. Ankylosing spondylitis and axial spondyloarthritis. N Engl J Med 2016;374:2563-74.

11. Ravelli A, Felici E, Magni-Manzoni S, Pistorio A, Novarini C, Bozzola E, et al. Patients with antinuclear antibody-positive juvenile idiopathic arthritis constitute a homogeneous subgroup irrespective of the course of joint disease. Arthritis Rheum 2005;52:826-32.

12. Ravelli A, Varnier GC, Oliveira S, Castell E, Arguedas O, Magnani A, et al. Antinuclear antibody-positive patients should be grouped as a separate category in the classification of juvenile idiopathic arthritis. Arthritis Rheum 2011;63:267-75.

13. Gregorio A, Gambini C, Gerloni V, Parafioriti A, Sormani MP, Gregorio S, et al. Lymphoid neogenesis in juvenile idiopathic arthritis correlates with ANA positivity and plasma cells infiltration. Rheumatology 2007;46:308-13.

14. Barnes MG, Grom AA, Thompson SD, Griffin TA, Pavlidis P, Itert L, et al. Subtype-specific peripheral blood gene expression profiles in recent-onset juvenile idiopathic arthritis. Arthritis Rheum 2009;60:2102-12.

15. Nordal E, Zak M, Aalto K, Berntson L, Fasth A, Herlin T, et al. Ongoing disease activity and changing categories in a long-term Nordic cohort study of juvenile idiopathic arthritis. Arthritis Rheum 2011;63:2809-18.

16. Guzman J, Henrey A, Loughin T, Berard RA, Shiff NJ, Jurencak R, et al. Predicting which children with juvenile idiopathic arthritis will have a severe disease course: results from the ReACCh-Out cohort. J Rheumatol 2017;44:230-40.

17. Glerup M, Herlin T, Twilt M. Remission rate is not dependent on the presence of antinuclear antibodies in juvenile idiopathic arthritis. Clin Rheumatol 2017;36:671-6.

18. Stoll ML, Zurakowski D, Nigrovic LE, Nichols DP, Sundel RP, Nigrovic PA. Patients with juvenile psoriatic arthritis comprise two distinct populations. Arthritis Rheum 2006;54:3564-72.

19. Stoll ML, Nigrovic PA, Gotte AC, Punaro M. Clinical comparison of early-onset psoriatic and non-psoriatic oligoarticular juvenile idiopathic arthritis. Clin Exp Rheumatol 2011;29:582-8.

20. Nigrovic PA. Juvenile psoriatic arthritis: bathwater or baby? J Rheumatol 2009;36:1861-3.

21. Taylor W, Gladman D, Helliwell P, Marchesoni A, Mease P, Mielants H. Classification criteria for psoriatic arthritis: development of new criteria from a large international study. Arthritis Rheum 2006;54:2665-73.

J Rheumatol 2019;46:124-6; doi:10.3899/jrheum.180922 